\title{
Existence and stability of asymmetric Burgers vortices
}

\author{
Thierry Gallay \\ Institut Fourier \\ Université de Grenoble I \\ BP 74 \\ 38402 Saint-Martin-d'Hères \\ France
}

\author{
C. Eugene Wayne \\ Department of Mathematics \\ and Center for BioDynamics \\ Boston University \\ 111 Cummington St. \\ Boston, MA 02215, USA
}

March 15, 2005

\begin{abstract}
Burgers vortices are stationary solutions of the three-dimensional Navier-Stokes equations in the presence of a background straining flow. These solutions are given by explicit formulas only when the strain is axisymmetric. In this paper we consider a weakly asymmetric strain and prove in that case that non-axisymmetric vortices exist for all values of the Reynolds number. In the limit of large Reynolds numbers, we recover the asymptotic results of Moffatt, Kida \& Ohkitani 11. We also show that the asymmetric vortices are stable with respect to localized two-dimensional perturbations.
\end{abstract}

\section{Introduction}

Localized structures such as vortex sheets or tubes play a prominent role in the dissipation of energy in three-dimensional turbulent flows. It is believed that these dissipative structures take place due to the interplay of two basic mechanisms: amplification of vorticity due to stretching, and diffusion through the action of viscosity [21]. A typical example that exhibits both features is the familiar Burgers vortex [1, an explicit solution of the three-dimensional Navier-Stokes equations in the presence of an axisymmetric background straining flow. In real flows, however, the local strain has no reason of being axisymmetric, and as a matter of fact the vortex tubes observed in numerical simulations usually exhibit a truly elliptical core region. It is therefore important to study the analogue of the Burgers vortex when the straining flow is asymmetric, although no explicit expression is available in that case.

Using a double series expansion, Robinson and Saffman [17] formally established the existence of an asymmetric vortex for small values of the Reynolds number $R$ and of the asymmetry parameter $\lambda$. This solution was also studied numerically for larger $\lambda$ (up to $3 / 4$ ) and $R$ (up to 100). On the other hand, an asymptotic expansion for large Reynolds numbers was performed by Moffatt, Kida and Ohkitani [11, see also [9]. Their results 
indicate that an equilibrium stretched vortex should exist for all values of $\lambda \in(0,1)$ and $R>0$ such that $\lambda / R \ll 1$. Interesting features of these solutions, such as the shape of isovorticity contours and the spatial distribution of energy dissipation, were also studied in detail. Finally, the stability of symmetric or non-symmetric vortices is an important issue which has attracted a lot of attention in recent years. Roughly speaking, the stability with respect to two-dimensional perturbations (i.e., perturbations which are independent of the axial coordinate) is well understood [17, 13, 14, 7, but only partial results have been obtained in the general case where arbitrary three-dimensional perturbations are allowed [18, 4, 5, 19, 8,

In this paper, we prove (rigorously) that non-axisymmetric Burgers vortices exist for all values of the Reynolds number, provided the asymmetry parameter is sufficiently small. In particular, taking the limit $R \rightarrow \infty$, we recover exactly the asymptotic results of Moffatt, Kida and Ohkitani [11. We also show that these vortices are stable with respect to spatially localized two-dimensional perturbations. Existence for larger values of the asymmetry parameter and stability with respect to three-dimensional perturbations are difficult questions, which have been solved so far for small Reynolds numbers only [8].

We now describe our results in more detail. We consider an incompressible viscous fluid filling the whole space $\mathbf{R}^{3}$, and we suppose that the velocity field is a two-dimensional perturbation of a linear straining flow, namely

$$
\mathbf{U}\left(x_{1}, x_{2}, x_{3}, t\right)=\left(\begin{array}{c}
\gamma_{1} x_{1} \\
\gamma_{2} x_{2} \\
\gamma_{3} x_{3}
\end{array}\right)+\left(\begin{array}{c}
u_{1}\left(x_{1}, x_{2}, t\right) \\
u_{2}\left(x_{1}, x_{2}, t\right) \\
0
\end{array}\right),
$$

where $\gamma_{1}, \gamma_{2}, \gamma_{3}$ are reals constants satisfying $\gamma_{1}+\gamma_{2}+\gamma_{3}=0$. Throughout this paper we assume that

$$
\gamma_{1}=-\frac{\gamma}{2}(1+\lambda), \quad \gamma_{2}=-\frac{\gamma}{2}(1-\lambda), \quad \gamma_{3}=\gamma,
$$

for some $\gamma>0$ and some $\lambda \in[0,1)$. Thus $\gamma_{3}$ is the only positive principal rate of strain, and the straining flow is axisymmetric if and only if $\lambda=0$. The case of a biaxial strain $(\lambda>1)$, which is also important for applications in turbulence, will not be considered here.

The vorticity $\Omega=\nabla \times \mathbf{U}$ is aligned with the vertical axis and depends only on the horizontal variable, namely

$$
\boldsymbol{\Omega}\left(x_{1}, x_{2}, x_{3}, t\right)=\left(\begin{array}{c}
0 \\
0 \\
\omega\left(x_{1}, x_{2}, t\right)
\end{array}\right) \text {, where } \omega=\partial_{1} u_{2}-\partial_{2} u_{1} .
$$

The evolution equation for $\omega$ reads

$$
\partial_{t} \omega+\left(u_{1}+\gamma_{1} x_{1}\right) \partial_{1} \omega+\left(u_{2}+\gamma_{2} x_{2}\right) \partial_{2} \omega=\nu \Delta \omega+\gamma_{3} \omega,
$$

where $\nu>0$ is the kinematic viscosity of the fluid. Since $\partial_{1} u_{1}+\partial_{2} u_{2}=0$ and $\partial_{1} u_{2}-\partial_{2} u_{1}=$ $\omega$, the rotational velocity $\mathbf{u}=\left(u_{1}, u_{2}\right)$ can be recovered from $\omega$ via the two-dimensional Biot-Savart law

$$
\mathbf{u}(x, t)=\frac{1}{2 \pi} \int_{\mathbf{R}^{2}} \frac{(x-y)^{\perp}}{|x-y|^{2}} \omega(y, t) \mathrm{d} y, \quad x=\left(x_{1}, x_{2}\right) \in \mathbf{R}^{2},
$$


where $x^{\perp}=\left(-x_{2}, x_{1}\right)$ and $|x|^{2}=x_{1}^{2}+x_{2}^{2}$.

In the axisymmetric case $\lambda=0$, equation (2) has a family of explicit time-independent solutions:

$$
\omega^{\Gamma}=\frac{\Gamma}{\delta^{2}} G\left(\frac{x}{\delta}\right), \quad \mathbf{u}^{\Gamma}=\frac{\Gamma}{\delta} \mathbf{v}^{G}\left(\frac{x}{\delta}\right)
$$

where $\Gamma \in \mathbf{R}, \delta=(\nu / \gamma)^{1 / 2}$, and

$$
G(x)=\frac{1}{4 \pi} e^{-|x|^{2} / 4}, \quad \mathbf{v}^{G}(x)=\frac{1}{2 \pi} \frac{x^{\perp}}{|x|^{2}}\left(1-e^{-|x|^{2} / 4}\right), \quad x \in \mathbf{R}^{2} .
$$

These are the well-known (axisymmetric) Burgers vortices. The family is indexed by the parameter $\Gamma=\int_{\mathbf{R}^{2}} \omega^{\Gamma} \mathrm{d} x$, which represents the circulation of $\mathbf{u}^{\Gamma}$ at infinity. The Reynolds number associated to the Burgers vortex with circulation $\Gamma$ can be defined [1] as

$$
R=\frac{|\Gamma|}{\nu}
$$

The aim of this paper is to study the analogue of the Burgers vortices when the straining flow is not axisymmetric. The expressions of these asymmetric vortices will be greatly simplified if we use the natural lengthscale $\delta=(\nu / \gamma)^{1 / 2}$ and timescale $\tau=1 / \gamma$ defined by the viscosity and the strain. We thus replace the variables $x, t$ and the functions $\mathbf{u}, \omega$ with the dimensionless quantities

$$
\tilde{x}=\frac{x}{\delta}, \quad \tilde{t}=\frac{t}{\tau}, \quad \tilde{\mathbf{u}}=\frac{\tau \mathbf{u}}{\delta}, \quad \tilde{\omega}=\tau \omega .
$$

Dropping the tildes for convenience, we see that the new functions $\omega, \mathbf{u}$ satisfy (2) with $\gamma=\nu=1$, namely

$$
\partial_{t} \omega+\mathbf{u} \cdot \nabla \omega=\mathcal{L} \omega+\lambda \mathcal{M} \omega
$$

where

$$
\mathcal{L}=\Delta+\frac{1}{2} x \cdot \nabla+1, \quad \mathcal{M}=\frac{1}{2}\left(x_{1} \partial_{1}-x_{2} \partial_{2}\right) .
$$

It is easily verified that $\mathcal{L} G=0$ and $\mathbf{v}^{G} \cdot \nabla G=0$, hence in the symmetric case $\lambda=0$ the Burgers vortex $\omega^{\alpha}=\alpha G, \mathbf{u}^{\alpha}=\alpha \mathbf{v}^{G}$ is indeed a stationary solution of (6) for any $\alpha \in \mathbf{R}$. The Reynolds number associated to this flow is simply $R=|\alpha|$.

To formulate our results, we introduce appropriate function spaces. Let $X$ be the (real) Hilbert space

$$
X=\left\{w \in L^{2}\left(\mathbf{R}^{2}\right) \mid G^{-1 / 2} w \in L^{2}\left(\mathbf{R}^{2}\right), \int_{\mathbf{R}^{2}} w \mathrm{~d} x=0\right\},
$$

equipped with the scalar product

$$
\left(w_{1}, w_{2}\right)_{X}=\int_{\mathbf{R}^{2}} G(x)^{-1} w_{1}(x) w_{2}(x) \mathrm{d} x .
$$

We also define the subspace $Y=\left\{w \in X \mid \partial_{i} w \in X\right.$ for $\left.i=1,2\right\}$ equipped with the natural scalar product

$$
\left(w_{1}, w_{2}\right)_{Y}=\int_{\mathbf{R}^{2}} G(x)^{-1}\left(w_{1}(x) w_{2}(x)+\nabla w_{1}(x) \cdot \nabla w_{2}(x)\right) \mathrm{d} x .
$$

Except for the zero mean condition, the space $X$ is just a weighted $L^{2}$ space (with Gaussian weight) and $Y$ is the corresponding Sobolev space. We can now state our first result: 
Theorem 1.1 There exist $\lambda_{0}>0$ and $K_{0}>0$ such that, for all $\lambda \in\left[0, \lambda_{0}\right]$ and all $\alpha \in \mathbf{R}$, equation (6) has a unique stationary solution $\omega^{\alpha, \lambda}, \mathbf{u}^{\alpha, \lambda}$ such that $\left\|\omega^{\alpha, \lambda}-\alpha G\right\|_{Y} \leq K_{0}$. Moreover, $\left\|\omega^{\alpha, \lambda}-\alpha G\right\|_{Y} \leq K_{0}\left(\lambda / \lambda_{0}\right)|\alpha| /(1+|\alpha|)$.

This theorem shows that, if the asymmetry parameter $\lambda$ is sufficiently small, equation (6) has a family of equilibria $\omega^{\alpha, \lambda}$ indexed by the circulation number $\alpha=\int_{\mathbf{R}^{2}} \omega^{\alpha, \lambda} \mathrm{d} x$. The solution $\omega^{\alpha, \lambda}$ is locally unique, has a Gaussian decay at infinity, and converges to the Burgers vortex $\alpha G$ as $\lambda \rightarrow 0$. Further properties of these asymmetric vortices will be established in Section 4. For instance, $\omega^{\alpha, \lambda}(x)$ is a smooth function of $x \in \mathbf{R}^{2}, \alpha \in \mathbf{R}$, and $\lambda \in\left[0, \lambda_{0}\right]$. Moreover, $\omega^{\alpha, \lambda}>0$ if $\alpha>0, \omega^{\alpha, \lambda}<0$ if $\alpha<0$, and $\omega^{\alpha, \lambda} \equiv 0$ if $\alpha=0$.

Theorem 1.1 will be proved by a classical perturbation argument. The only remarkable point is that this argument can be applied uniformly for all $\alpha \in \mathbf{R}$. In particular, for fixed $\lambda$, we can investigate the limit of large Reynolds numbers $R=|\alpha| \rightarrow \infty$. The last inequality in the theorem asserts that $\alpha^{-1} \omega^{\alpha, \lambda}=G+\mathcal{O}(\lambda / R)$ as $R \rightarrow \infty$, hence we recover the observation by Moffatt, Kida and Ohkitani [11] that the asymptotic profile is always the Gaussian $G$, even in the asymmetric case $\lambda>0$. (Note that, unlike in [1], we do not need to assume that the asymptotic profile is radially symmetric.) Moreover, the deviation from the limiting profile is proportional to $\lambda / R$ at leading order, as established in 11. A rigorous expansion up to second order in $\lambda$ and $R^{-1}$ will be performed in Section 4, see Eq.(38) below.

Our second result shows that the asymmetric Burgers vortex is asymptotically stable stable with respect to perturbations in $X$. As in Theorem 1.1 this property holds uniformly for all $\alpha \in \mathbf{R}$ and $\lambda \in\left[0, \lambda_{1}\right]$, for some $\lambda_{1}>0$ (possibly smaller than $\lambda_{0}$ ). Remark that there is no loss of generality in assuming that the perturbations have zero mean, because if $\int \tilde{\omega} \mathrm{d} x=\beta \neq 0$ then $\omega^{\alpha, \lambda}+\tilde{\omega}$ is a zero mean perturbation of the (modified) $\operatorname{vortex} \omega^{\alpha+\beta, \lambda}$.

Theorem 1.2 Given any $\mu \in(0,1 / 2)$, there exist $\lambda_{1}>0$ and $\varepsilon>0$ such that, for all $\lambda \in\left[0, \lambda_{1}\right]$ and all $\alpha \in \mathbf{R}$, the following holds. For all initial data $\omega_{0}$ with $\left\|\omega_{0}-\omega^{\alpha, \lambda}\right\|_{X} \leq \varepsilon$, equation (6) has a unique global solution $\omega(x, t)$ such that $\omega-\omega^{\alpha, \lambda} \in C^{0}([0,+\infty), X)$. Moreover,

$$
\left\|\omega(\cdot, t)-\omega^{\alpha, \lambda}\right\|_{X} \leq\left\|\omega_{0}-\omega^{\alpha, \lambda}\right\|_{X} e^{-\mu t}, \quad \text { for all } t \geq 0
$$

The proof of Theorem 1.1 is based on the following ideas. Given $\lambda \in(0,1)$ and $\alpha \in \mathbf{R}$, we look for stationary solutions of (6) of the form $\omega=\alpha G+w, \mathbf{u}=\alpha \mathbf{v}^{G}+\mathbf{v}$, where $\int_{\mathbf{R}^{2}} w \mathrm{~d} x=0$ and $\mathbf{v}$ is the velocity field obtained from $w$ via the Biot-Savart law (3). The equation for $w$ reads:

$$
\alpha\left(\mathbf{v}^{G} \cdot \nabla w+\mathbf{v} \cdot \nabla G\right)+\mathbf{v} \cdot \nabla w=\mathcal{L} w+\lambda \mathcal{M}(\alpha G+w) .
$$

Let $\Lambda$ be the integro-differential operator defined by

$$
\Lambda w=\mathbf{v}^{G} \cdot \nabla w+\mathbf{v} \cdot \nabla G .
$$

It is shown in [7] that, for any $\alpha \in \mathbf{R}$, the spectrum of $\mathcal{L}-\alpha \Lambda$ acting on $X$ is contained in the half-plane $\left\{z \in \mathbf{C} \mid \operatorname{Re}(z) \leq-\frac{1}{2}\right\}$. In particular $\mathcal{L}-\alpha \Lambda$ is invertible, and (10) can be rewritten as

$$
w=(\mathcal{L}-\alpha \Lambda)^{-1}(\mathbf{v} \cdot \nabla w-\lambda \mathcal{M}(\alpha G+w)) .
$$


In Section 2] we show that $(\mathcal{L}-\alpha \Lambda)^{-1} \mathcal{M}$ is a bounded operator in $Y$ whose norm is uniformly bounded for all $\alpha \in \mathbf{R}$. In Section 3. we prove that the function $w_{\alpha} \in Y$ defined by

$$
w_{\alpha}=-\alpha(\mathcal{L}-\alpha \Lambda)^{-1} \mathcal{M} G
$$

is uniformly bounded in $Y$ for all $\alpha \in \mathbf{R}$, and converges to some limit $w_{\infty}$ as $|\alpha| \rightarrow \infty$. After these preliminaries, a standard contraction argument allows to prove that (12) has a unique solution $w^{\alpha, \lambda}$ (in an appropriate ball in $Y$ ) if $\lambda$ is sufficiently small, and that $w^{\alpha, \lambda}=\lambda w_{\alpha}+\mathcal{O}\left(\lambda^{2}\right)$. This is done in Section 4, where additional properties of the asymmetric Burgers vortex $\omega^{\alpha, \lambda}=\alpha G+w^{\alpha, \lambda}$ are also established. Theorem 1.2 is proved in Section 5 by an energy estimate, using the observation that the linearization of (6) at the vortex $\omega^{\alpha, \lambda}$ is a small perturbation of the linear equation $\partial_{t} \omega=(\mathcal{L}-\alpha \Lambda) \omega$ if $\lambda$ is small.

\section{Linearization in the symmetric case}

If $\mathcal{L}, \Lambda$ are the linear operators defined in (7), (11), we know from [7] that $\mathcal{L}-\alpha \Lambda$ is invertible in $X$ (with bounded inverse) for all $\alpha \in \mathbf{R}$. In this section we use the methods of [7] to establish the following result:

Proposition 2.1 There exist positive constants $K_{1}, K_{2}$ such that, for all $\alpha \in \mathbf{R}$, the following inequalities hold:

$$
\begin{aligned}
\left\|(\mathcal{L}-\alpha \Lambda)^{-1} w\right\|_{Y} & \leq K_{1}\|w\|_{X}, \quad \text { for all } w \in X \\
\left\|(\mathcal{L}-\alpha \Lambda)^{-1} \mathcal{M} w\right\|_{Y} & \leq K_{2}\|w\|_{Y}, \quad \text { for all } w \in Y
\end{aligned}
$$

Proof: The properties of the linear operator $\mathcal{L}$ acting on $X$ are easy to establish, because this operator is conjugated to the Hamiltonian of the harmonic oscillator in $\mathbf{R}^{2}$. Let $H$ be the closed subspace of $L^{2}\left(\mathbf{R}^{2}\right)$ defined by $H=\left\{f \in L^{2}\left(\mathbf{R}^{2}\right) \mid \int_{\mathbf{R}^{2}} G^{1 / 2} f \mathrm{~d} x=0\right\}$. Consider the linear operator $L: D(L) \rightarrow H$ defined by

$$
\begin{aligned}
D(L) & =\left\{\left.f \in H\left|\Delta f \in L^{2}\left(\mathbf{R}^{2}\right),\right| x\right|^{2} f \in L^{2}\left(\mathbf{R}^{2}\right)\right\} \\
L & =G^{-1 / 2}(-\mathcal{L}) G^{1 / 2}=-\Delta+\frac{|x|^{2}}{16}-\frac{1}{2} .
\end{aligned}
$$

As is well-known, $L$ is self-adjoint in $H$ with spectrum $\sigma(L)=\{n / 2 \mid n=1,2,3, \ldots\}$. In particular, $L \geq 1 / 2$. It follows that there exists $C_{1}>0$ such that, for all $f \in H$,

$$
\left\|L^{-1 / 2} f\right\|_{L^{2}}+\left\||x| L^{-1 / 2} f\right\|_{L^{2}}+\left\|\nabla L^{-1 / 2} f\right\|_{L^{2}} \leq C_{1}\|f\|_{L^{2}} .
$$

Indeed, setting $g=L^{-1 / 2} f$, we have

$$
\begin{aligned}
\|f\|_{L^{2}}^{2} & =\left\|L^{1 / 2} g\right\|_{L^{2}}^{2}=(g, L g)_{L^{2}} \\
& =\frac{1}{4} \int_{\mathbf{R}^{2}}\left(|\nabla g|^{2}+\frac{|x|^{2}}{16} g^{2}-\frac{1}{2} g^{2}\right) \mathrm{d} x+\frac{3}{4}(g, L g)_{L^{2}} \\
& \geq \frac{1}{4} \int_{\mathbf{R}^{2}}\left(|\nabla g|^{2}+\frac{|x|^{2}}{16} g^{2}+g^{2}\right) \mathrm{d} x,
\end{aligned}
$$


where we have used the fact that $(g, L g)_{L^{2}} \geq \frac{1}{2}\|g\|_{L^{2}}^{2}$. The bounds (16) also show that $L^{-1 / 2}$ is a compact operator in $H$.

Another useful estimate can be obtained from (16) by a duality argument. For $j=1$ or 2, let $H_{j}=\left\{f \in H \mid \int_{\mathbf{R}^{2}} x_{j} G^{1 / 2} f \mathrm{~d} x=0\right\}$. Then $L^{-1 / 2} x_{j}$ extends to a bounded operator from $H_{j}$ into $H$, and for all $f \in H_{j}$ we have

$$
\left\|L^{-1 / 2} x_{j} f\right\|_{L^{2}} \leq C_{1}\|f\|_{L^{2}} .
$$

Indeed, by density, it is sufficient to prove (18) for $f \in H_{j} \cap \mathcal{S}\left(\mathbf{R}^{2}\right)$, where $\mathcal{S}$ denotes the Schwartz space of test functions. In that case $x_{j} f \in H$ and for all $\varphi \in H$ we have

$$
\left|\left(\varphi, L^{-1 / 2} x_{j} f\right)_{L^{2}}\right|=\left|\left(x_{j} L^{-1 / 2} \varphi, f\right)_{L^{2}}\right| \leq\left\|x_{j} L^{-1 / 2} \varphi\right\|_{L^{2}}\|f\|_{L^{2}} \leq C_{1}\|\varphi\|_{L^{2}}\|f\|_{L^{2}},
$$

which proves (18).

We now return to the operator $\mathcal{L}: D(\mathcal{L}) \rightarrow X$ defined by $D(\mathcal{L})=G^{1 / 2} D(L), X=$ $G^{1 / 2} H$, and $-\mathcal{L}=G^{1 / 2} L G^{-1 / 2}$. By construction, $\mathcal{L}$ is selfadjoint in $X,-\mathcal{L} \geq 1 / 2$, and $(-\mathcal{L})^{-1 / 2}$ is a compact operator in $X$. Using (16), (18) we easily obtain the following additional properties:

\section{Lemma 2.2}

i) $(-\mathcal{L})^{-1 / 2}$ is a bounded operator from $X$ into $Y$;

ii) $(-\mathcal{L})^{-1 / 2} \mathcal{M}$ extends to a bounded operator from $Y$ into $X$.

\section{Proof of Lemma 2.2.}

i) Let $w \in X$. Since $(-\mathcal{L})^{-1 / 2}=G^{1 / 2} L^{-1 / 2} G^{-1 / 2}$, we have

$$
\begin{aligned}
\left\|(-\mathcal{L})^{-1 / 2} w\right\|_{X} & =\left\|G^{-1 / 2}(-\mathcal{L})^{-1 / 2} w\right\|_{L^{2}}=\left\|L^{-1 / 2} G^{-1 / 2} w\right\|_{L^{2}} \\
& \leq C_{1}\left\|G^{-1 / 2} w\right\|_{L^{2}}=C_{1}\|w\|_{X} .
\end{aligned}
$$

Similarly, since $\nabla G^{1 / 2}=-\frac{x}{4} G^{1 / 2}$, we find

$$
\begin{aligned}
\left\|\nabla(-\mathcal{L})^{-1 / 2} w\right\|_{X} & =\left\|G^{-1 / 2} \nabla G^{1 / 2} L^{-1 / 2} G^{-1 / 2} w\right\|_{L^{2}} \\
& \leq\left\|\nabla L^{-1 / 2} G^{-1 / 2} w\right\|_{L^{2}}+\frac{1}{4}\left\||x| L^{-1 / 2} G^{-1 / 2} w\right\|_{L^{2}} \\
& \leq C_{1}\left\|G^{-1 / 2} w\right\|_{L^{2}}=C_{1}\|w\|_{X} .
\end{aligned}
$$

ii) For any $w \in Y$, we have $\left\|(-\mathcal{L})^{-1 / 2} \mathcal{M} w\right\|_{X}=\frac{1}{2}\left\|L^{-1 / 2} G^{-1 / 2}\left(x_{1} \partial_{1}-x_{2} \partial_{2}\right) w\right\|_{L^{2}}$. Now $G^{-1 / 2} \partial_{j} w \in H_{j}$ for $j=1,2$, hence by (18)

$$
\left\|L^{-1 / 2} x_{j} G^{-1 / 2} \partial_{j} w\right\|_{L^{2}} \leq C_{1}\left\|G^{-1 / 2} \partial_{j} w\right\|_{L^{2}} \leq C_{1}\|w\|_{Y} .
$$

We conclude that $\left\|(-\mathcal{L})^{-1 / 2} \mathcal{M} w\right\|_{X} \leq C_{1}\|w\|_{Y}$.

Finally, we consider the operator $\Sigma=(-\mathcal{L})^{-1 / 2} \Lambda(-\mathcal{L})^{-1 / 2}$, where $\Lambda$ is defined by (11). The following properties of $\Sigma$ will be useful:

\section{Lemma 2.3}

i) The operator $\Sigma$ is compact in $X$;

ii) The operator $\Sigma$ is skew-symmetric in $X$;

iii) For any $\alpha \in \mathbf{R}$, the operator $\mathbf{1}+\alpha \Sigma$ is invertible in $X$ and

$$
\left\|(\mathbf{1}+\alpha \Sigma)^{-1} w\right\|_{X} \leq\|w\|_{X}, \quad \text { for all } w \in X .
$$




\section{Proof of Lemma 2.3,}

i) Since $(-\mathcal{L})^{-1 / 2}$ is compact in $X$ and bounded from $X$ into $Y$, it suffices to show that $\Lambda: Y \rightarrow X$ is bounded. If $w \in Y$, then

$$
\left\|\mathbf{v}^{G} \cdot \nabla w\right\|_{X} \leq\left\|\mathbf{v}^{G}\right\|_{L^{\infty}}\|\nabla w\|_{X} \leq C\|w\|_{Y} .
$$

On the other hand, by Hölder's inequality, we have for all $p \in[1,2]$ :

$$
\|w\|_{L^{p}}=\left\|G^{1 / 2} G^{-1 / 2} w\right\|_{L^{p}} \leq\left\|G^{1 / 2}\right\|_{L^{\frac{2 p}{2-p}}}\left\|G^{-1 / 2} w\right\|_{L^{2}}=C\|w\|_{X} .
$$

If $\mathbf{v}$ denotes the velocity field obtained from $w$ via the Biot-Savart law (3), the HardyLittlewood-Sobolev inequality [10] implies that $\mathbf{v} \in L^{q}\left(\mathbf{R}^{2}\right)$ for all $q \in(2, \infty)$, and the following bounds hold:

$$
\|\mathbf{v}\|_{L^{q}} \leq C_{p}\|w\|_{L^{p}}, \quad \text { where } \quad 1<p<2 \quad \text { and } \quad \frac{1}{q}=\frac{1}{p}-\frac{1}{2} .
$$

Thus

$$
\|\mathbf{v} \cdot \nabla G\|_{X}=\left\|G^{-1 / 2} \mathbf{v} \cdot \nabla G\right\|_{L^{2}} \leq\|\mathbf{v}\|_{L^{4}}\left\|G^{-1 / 2} \nabla G\right\|_{L^{4}} \leq C\|w\|_{L^{4 / 3}} \leq C\|w\|_{X} .
$$

We conclude that $\|\Lambda w\|_{X} \leq C\|w\|_{Y}$.

ii) It is shown in ([7], Lemma 4.8) that $\left(w_{1}, \Lambda w_{2}\right)_{X}+\left(\Lambda w_{1}, w_{2}\right)_{X}=0$ for all $w_{1}, w_{2} \in Y$. Since $(-\mathcal{L})^{-1 / 2}$ is symmetric in $X$, it follows that $\left(w_{1}, \Sigma w_{2}\right)_{X}+\left(\Sigma w_{1}, w_{2}\right)_{X}=0$ for all $w_{1}, w_{2} \in X$.

iii) By the analytic Fredholm theorem [16], we know that $1+\alpha \Sigma$ is invertible in $X$ for all $\alpha \in \mathbf{R}$ except perhaps on a discrete set (with no limit point) where the meromorphic map $\alpha \mapsto(\mathbf{1}+\alpha \Sigma)^{-1}$ has poles. But whenever $\mathbf{1}+\alpha \Sigma$ is invertible we have by ii):

$$
\|w\|_{X}^{2}=(w,(\mathbf{1}+\alpha \Sigma) w)_{X} \leq\|w\|_{X}\|(\mathbf{1}+\alpha \Sigma) w\|_{X} \text { for all } w \in X,
$$

hence $\left\|(\mathbf{1}+\alpha \Sigma)^{-1} w\right\|_{X} \leq\|w\|_{X}$. This implies that $\mathbf{1}+\alpha \Sigma$ is invertible for all $\alpha \in \mathbf{R}$ and that (19) holds.

Equipped with these lemmas, it is now straightforward to conclude the proof of Proposition 2.1. For any $\alpha \in \mathbf{R}$, the formula

$$
(\mathcal{L}-\alpha \Lambda)^{-1}=-(-\mathcal{L})^{-1 / 2}(\mathbf{1}+\alpha \Sigma)^{-1}(-\mathcal{L})^{-1 / 2}
$$

shows that $\mathcal{L}-\alpha \Lambda$ is invertible in $X$ for all $\alpha \in \mathbf{R}$. The bounds (14) and (15) are then direct consequences of this identity and Lemmas 2.2. 2.3.

\section{$3 \quad$ Large Reynolds number asymptotics}

The main goal of this section is to prove that the function $w_{\alpha}$ defined by (13) is uniformly bounded in the space $Y$ for all $\alpha \in \mathbf{R}$. From (13) we expect that $w_{\alpha} \rightarrow \Lambda^{-1} \mathcal{M} G$ as $|\alpha| \rightarrow \infty$, but it is not clear a priori that this limit makes sense because $\Lambda$ is not an invertible operator. Our first result shows that $\mathcal{M} G$ is indeed in the range of $\Lambda$ (we recall that $\mathcal{S}\left(\mathbf{R}^{2}\right)$ is the Schwartz space of test functions): 
Proposition 3.1 There exists $w_{\infty} \in \mathcal{S}\left(\mathbf{R}^{2}\right)$ such that $\Lambda w_{\infty}=\mathcal{M} G$.

Proof: By (5), (7) we have $\mathcal{M} G=\frac{1}{2}\left(x_{1} \partial_{1}-x_{2} \partial_{2}\right) G=-\frac{1}{4}\left(x_{1}^{2}-x_{2}^{2}\right) G$. Using polar coordinates in $\mathbf{R}^{2}$, we thus find

$$
(\mathcal{M} G)(r \cos \theta, r \sin \theta)=-\frac{1}{4} r^{2} g(r) \cos (2 \theta)
$$

where $g(r)=G(r \cos \theta, r \sin \theta)=(4 \pi)^{-1} e^{-r^{2} / 4}$. As was observed in [17, 11, 13, 7], the operator $\Lambda$ is invariant under rotations in the plane, and is therefore block-diagonal in the Fourier basis $\left\{e^{i n \theta}\right\}_{n \in \mathbf{Z}}$. We make the following Ansatz:

$$
w_{\infty}(r \cos \theta, r \sin \theta)=\omega(r) \sin (2 \theta),
$$

where $\omega: \mathbf{R}_{+} \rightarrow \mathbf{R}$ has to be determined. The velocity field associated to $w_{\infty}$ reads

$$
\mathbf{v}_{\infty}=\frac{2}{r} \Omega(r) \cos (2 \theta) \mathbf{e}_{r}-\Omega^{\prime}(r) \sin (2 \theta) \mathbf{e}_{\theta}
$$

where $\mathbf{e}_{r}$ is the unit vector in the radial direction, $\mathbf{e}_{\theta}=\mathbf{e}_{r}^{\perp}$, and where $\Omega: \mathbf{R}_{+} \rightarrow \mathbf{R}$ is the solution of the ordinary differential equation

$$
-\frac{1}{r}\left(r \Omega^{\prime}(r)\right)^{\prime}+\frac{4}{r^{2}} \Omega(r)=\omega(r), \quad r>0,
$$

which satisfies the boundary conditions $\Omega(0)=\Omega(+\infty)=0$. Using these expressions, we find

$$
\begin{aligned}
\left(\Lambda w_{\infty}\right)(r \cos \theta, r \sin \theta) & =\left(\mathbf{v}^{G} \cdot \nabla w_{\infty}+\mathbf{v}_{\infty} \cdot \nabla G\right)(r \cos \theta, r \sin \theta) \\
& =\cos (2 \theta)(2 \varphi(r) \omega(r)-g(r) \Omega(r)),
\end{aligned}
$$

where $\varphi(r)=\left(2 \pi r^{2}\right)^{-1}\left(1-e^{-r^{2} / 4}\right)$. If we compare (22) and (25) we obtain the solution

$$
\omega(r)=h(r)\left(\Omega(r)-\frac{r^{2}}{4}\right)
$$

where $h=g /(2 \varphi)$, i.e. $h(r)=\left(r^{2} / 4\right)\left(e^{r^{2} / 4}-1\right)^{-1}$. Inserting (26) into (24), we see that $\Omega$ should satisfy the ordinary differential equation

$$
-\frac{1}{r}\left(r \Omega^{\prime}(r)\right)^{\prime}+\left(\frac{4}{r^{2}}-h(r)\right) \Omega(r)=-\frac{r^{2} h(r)}{4}, \quad r>0
$$

together with the boundary conditions $\Omega(0)=\Omega(+\infty)=0$.

Remark 3.2 Equation 27) was derived and studied numerically by Moffatt, Kida and Ohkitani, see Eq.(2.25) in [11]. The notation used in [11] is $f=\Omega$ and $\eta=-h$.

To solve (27), we first consider the associated homogeneous equation

$$
-\frac{1}{r}\left(r \Omega^{\prime}(r)\right)^{\prime}+\left(\frac{4}{r^{2}}-h(r)\right) \Omega(r)=0, \quad r>0 .
$$


Setting $r=e^{ \pm t}$ and $\Omega(r)=F( \pm \log (r))$, this equation is transformed into

$$
-F^{\prime \prime}(t)+\left(4-H_{ \pm}(t)\right) F(t)=0, \quad t \in \mathbf{R}
$$

where $H_{ \pm}(t)=e^{ \pm 2 t} h\left(e^{ \pm t}\right)$. In particular, $H_{ \pm}(t)$ decays rapidly to zero as $t \rightarrow+\infty$. Applying Theorem 3.8.1 in [3], we deduce that (29) has a unique solution $F_{ \pm}(t)$ such that

$$
\lim _{t \rightarrow+\infty} e^{2 t}\left(\begin{array}{c}
F_{ \pm}(t) \\
F_{ \pm}^{\prime}(t)
\end{array}\right)=\left(\begin{array}{c}
1 \\
-2
\end{array}\right)
$$

We now define $\psi_{+}(r)=F_{+}(\log (r))$ and $\psi_{-}(r)=F_{-}(-\log (r))$. By construction, $\psi_{+}, \psi_{-}$are the only solutions of (28) such that

$$
\psi_{+}(r) \sim \frac{1}{r^{2}} \quad \text { as } r \rightarrow+\infty, \quad \psi_{-}(r) \sim r^{2} \quad \text { as } r \rightarrow 0 .
$$

We observe that the "potential" $4 / r^{2}-h(r)$ in (28) is strictly positive, because

$$
\inf _{r>0}\left(\frac{4}{r^{2}}-h(r)\right)=\inf _{z>0}\left(\frac{1}{z}-\frac{z}{e^{z}-1}\right)>0 .
$$

By the Maximum Principle [15], it follows that $\psi_{-}^{\prime}(r)>0$ and $\psi_{+}^{\prime}(r)<0$ for all $r>0$. In particular, $\psi_{+}$and $\psi_{-}$are linearly independent, hence there exists $w_{0}>0$ such that

$$
W(r)=\psi_{+}(r) \psi_{-}^{\prime}(r)-\psi_{+}^{\prime}(r) \psi_{-}(r)=\frac{w_{0}}{r}, \quad r>0
$$

Moreover,

$$
\psi_{+}(r) \sim \frac{w_{0}}{4 r^{2}} \quad \text { as } r \rightarrow 0, \quad \psi_{-}(r) \sim \frac{w_{0} r^{2}}{4} \quad \text { as } r \rightarrow+\infty
$$

Using these notations and the "variation of constants" formula, we obtain the following expression for the solution of (27):

$$
\Omega(r)=-\psi_{+}(r) \int_{0}^{r} \frac{z^{3}}{4 w_{0}} \psi_{-}(z) h(z) \mathrm{d} z-\psi_{-}(r) \int_{r}^{\infty} \frac{z^{3}}{4 w_{0}} \psi_{+}(z) h(z) \mathrm{d} z, \quad r>0 .
$$

It is clear that $\Omega: \mathbf{R}_{+} \rightarrow \mathbf{R}$ is a smooth function satisfying

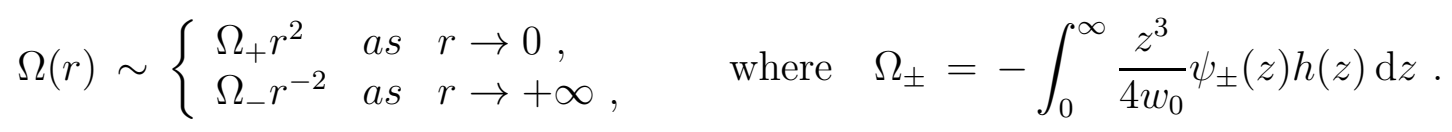

(The values $\Omega_{+} \approx-0.38$ and $\Omega_{-} \approx-17.5$ were found numerically in [11.) Similar estimates hold for all derivatives. Going back to (26) , we see that $\omega: \mathbf{R}_{+} \rightarrow \mathbf{R}$ is smooth and rapidly decreasing at infinity. Moreover $\omega(0)=0, \omega(r)<0$ for all $r>0$, and it is easy to verify that the Taylor expansion of $\omega(r)$ at $r=0$ contains even powers of $r$ only. Thus the function the function $w_{\infty}: \mathbf{R}^{2} \rightarrow \mathbf{R}$ defined by (23) is smooth, rapidly decreasing at infinity, and satisfies $\Lambda w_{\infty}=\mathcal{M} G$ by construction.

As a consequence we can prove that the function $w_{\alpha}$ defined in (13) is uniformly bounded in $Y$ : 
Corollary 3.3 There exists $K_{3}>0$ such that $\left\|w_{\alpha}\right\|_{Y} \leq K_{3}|\alpha| /(1+|\alpha|)$ for all $\alpha \in \mathbf{R}$.

Proof: From (13) and (15) we know that $\left\|w_{\alpha}\right\|_{Y} \leq K_{2}|\alpha|\|G\|_{Y}$. On the other hand, in view of Proposition 3.1. we have $(\mathcal{L}-\alpha \Lambda) w_{\alpha}=-\alpha \mathcal{M} G=(\mathcal{L}-\alpha \Lambda) w_{\infty}-\mathcal{L} w_{\infty}$, hence

$$
w_{\alpha}=w_{\infty}-(\mathcal{L}-\alpha \Lambda)^{-1} \mathcal{L} w_{\infty} .
$$

Using (14), we infer that $\left\|w_{\alpha}\right\|_{Y} \leq\left\|w_{\infty}\right\|_{Y}+K_{1}\left\|\mathcal{L} w_{\infty}\right\|_{X}$. Combining both estimates we obtain the desired result.

A more detailed analysis reveals that $w_{\infty}$ is indeed the limit of $w_{\alpha}$ as $|\alpha| \rightarrow \infty$ :

Proposition 3.4 There exists $K_{4}>0$ such that $\left\|w_{\alpha}-w_{\infty}\right\|_{Y} \leq K_{4} /(1+|\alpha|)$ for all $\alpha \in \mathbf{R}$.

Proof: By (31) and (14), we have $\left\|w_{\alpha}-w_{\infty}\right\|_{Y} \leq K_{1}\left\|\mathcal{L} w_{\infty}\right\|_{X}$. On the other hand, proceeding exactly as in the proof of Proposition 3.1. it is straightforward to show that there exists $z_{\infty} \in \mathcal{S}\left(\mathbf{R}^{2}\right)$ such that $\Lambda z_{\infty}=\mathcal{L} w_{\infty}$ (the details are left to the reader). Then, using (31), we find for all $\alpha \neq 0$

$$
w_{\alpha}=w_{\infty}+\frac{1}{\alpha} z_{\infty}-\frac{1}{\alpha}(\mathcal{L}-\alpha \Lambda)^{-1} \mathcal{L} z_{\infty},
$$

hence $\left\|w_{\alpha}-w_{\infty}\right\|_{Y} \leq|\alpha|^{-1}\left(\left\|z_{\infty}\right\|_{Y}+K_{1}\left\|\mathcal{L} z_{\infty}\right\|_{X}\right)$. Combining both estimates we obtain the desired result.

\section{Existence of asymmetric vortices}

As is explained in the introduction, we shall prove the existence of a stationary solution of (16) by solving Eq.(12), namely

$$
w=\lambda w_{\alpha}+(\mathcal{L}-\alpha \Lambda)^{-1}(\mathbf{v} \cdot \nabla w-\lambda \mathcal{M} w),
$$

where $w_{\alpha}$ is defined in (13) and (as usual) $\mathbf{v}$ denotes the velocity field obtained from $w$ by the Biot-Savart law (31). To bound the nonlinear term in (321), we use the following bilinear estimate:

Lemma 4.1 There exists $K_{5}>0$ such that, if $w, \tilde{w} \in Y$ and if $\mathbf{v}$ is the velocity field obtained from $w$ by the Biot-Savart law, then $\mathbf{v} \cdot \nabla \tilde{w} \in X$ and

$$
\|\mathbf{v} \cdot \nabla \tilde{w}\|_{X} \leq K_{5}\|w\|_{Y}\|\tilde{w}\|_{Y} .
$$

Proof: We first observe that $w \in L^{p}\left(\mathbf{R}^{2}\right)$ for all $p \in[1,+\infty)$, and that $\|w\|_{L^{p}} \leq C_{p}\|w\|_{Y}$. Indeed this is proved in (20) if $p \in[1,2]$, and if $p \geq 2$ this follows from the embeddings $Y \hookrightarrow H^{1}\left(\mathbf{R}^{2}\right) \hookrightarrow L^{p}\left(\mathbf{R}^{2}\right)$. Next, by the Calderón-Zygmund inequality [20], the velocity 
field $\mathbf{v}$ associated to $w$ satisfies $\|\nabla \mathbf{v}\|_{L^{p}} \leq C_{p}\|w\|_{L^{p}}$ for $1<p<\infty$. Using the GagliardoNirenberg inequality [12], we deduce that $\mathbf{v} \in L^{\infty}\left(\mathbf{R}^{2}\right)$ and that

$$
\|\mathbf{v}\|_{L^{\infty}} \leq C\|\nabla \mathbf{v}\|_{L^{3}}^{1 / 2}\|\mathbf{v}\|_{L^{6}}^{1 / 2} \leq C\|w\|_{L^{3}}^{1 / 2}\|w\|_{L^{3 / 2}}^{1 / 2} \leq C\|w\|_{Y},
$$

where we also used the Hardy-Littlewood-Sobolev inequality (21) with $q=6, p=3 / 2$. We conclude that $\mathbf{v} \cdot \nabla \tilde{w} \in X$ and

$$
\|\mathbf{v} \cdot \nabla \tilde{w}\|_{X} \leq\|\mathbf{v}\|_{L^{\infty}}\|\nabla \tilde{w}\|_{X} \leq C\|w\|_{Y}\|\tilde{w}\|_{Y},
$$

which is the desired result.

We can now prove the main result of this section:

Proposition 4.2 Choose $\lambda_{0} \in(0,1)$ such that

$$
2 K_{2} \lambda_{0}<1 \text {, and } 16 K_{1} K_{3} K_{5} \lambda_{0} \leq 1 .
$$

Then, for all $\lambda \in\left[0, \lambda_{0}\right]$ and all $\alpha \in \mathbf{R}$, equation (32) has a unique solution $w^{\alpha, \lambda} \in Y$ such that $\left\|w^{\alpha, \lambda}\right\|_{Y} \leq\left(4 K_{1} K_{5}\right)^{-1}$. This solution depends smoothly on the parameters $(\alpha, \lambda) \in \mathbf{R} \times\left[0, \lambda_{0}\right]$ and satisfies $\left\|w^{\alpha, \lambda}\right\|_{Y} \leq 4 K_{3} \lambda|\alpha| /(1+|\alpha|)$.

Proof: Fix $\lambda \in\left[0, \lambda_{0}\right], \alpha \in \mathbf{R}$, and choose $r>0$ such that

$$
4 K_{3}^{\alpha} \lambda \leq r \leq \frac{1}{4 K_{1} K_{5}}, \quad \text { where } \quad K_{3}^{\alpha}=\frac{K_{3}|\alpha|}{1+|\alpha|} .
$$

Let $F^{\alpha, \lambda}: Y \rightarrow Y$ be the quadratic map defined by

$$
F^{\alpha, \lambda}(w)=\lambda w_{\alpha}+(\mathcal{L}-\alpha \Lambda)^{-1}(\mathbf{v} \cdot \nabla w-\lambda \mathcal{M} w), \quad w \in Y .
$$

Using Proposition 2.1. Corollary 3.3 and Lemma 4.1, we find for all $w \in Y$ :

$$
\left\|F^{\alpha, \lambda}(w)\right\|_{Y} \leq K_{3}^{\alpha} \lambda+K_{1} K_{5}\|w\|_{Y}^{2}+K_{2} \lambda\|w\|_{Y} .
$$

Similarly, for all $w, \tilde{w} \in Y$ :

$$
\left\|F^{\alpha, \lambda}(w)-F^{\alpha, \lambda}(\tilde{w})\right\|_{Y} \leq\left(K_{1} K_{5}\left(\|w\|_{Y}+\|\tilde{w}\|_{Y}\right)+K_{2} \lambda\right)\|w-\tilde{w}\|_{Y} .
$$

Let $B_{r}=\left\{w \in Y \mid\|w\|_{Y} \leq r\right\}$. It follows from (33), (34), and (35) that $F^{\alpha, \lambda}$ maps $B_{r}$ into itself, because $K_{3}^{\alpha} \lambda+K_{1} K_{5} r^{2}+K_{2} \lambda r \leq r / 4+r / 4+r / 2=r$. Similarly (36) implies for all $w, \tilde{w} \in B_{r}$ :

$$
\left\|F^{\alpha, \lambda}(w)-F^{\alpha, \lambda}(\tilde{w})\right\|_{Y} \leq \kappa\|w-\tilde{w}\|_{Y}, \quad \text { where } \quad \kappa=\frac{1}{2}+K_{2} \lambda_{0}<1 .
$$

By the contraction mapping theorem, $F^{\alpha, \lambda}$ has thus a unique fixed point in $B_{r}$, which we denote by $w^{\alpha, \lambda}$. It remains to show that $w^{\alpha, \lambda}$ is a smooth function of $(\alpha, \lambda) \in \mathbf{R} \times\left[0, \lambda_{0}\right]$. But this is a direct consequence of the implicit function theorem, because the map $F^{\alpha, \lambda}$ depends smoothly on $(\alpha, \lambda)$ and the differential

$$
D_{w} F^{\alpha, \lambda}(w)=\tilde{w} \mapsto(\mathcal{L}-\alpha \Lambda)^{-1}(\tilde{\mathbf{v}} \cdot \nabla w+\mathbf{v} \cdot \nabla \tilde{w}-\lambda \mathcal{M} \tilde{w})
$$


satisfies $\left\|D_{w} F^{\alpha, \lambda}(w)\right\| \leq \kappa$ for all $w \in B_{r}$ and all $(\alpha, \lambda) \in \mathbf{R} \times\left[0, \lambda_{0}\right]$. Thus $\mathbf{1}-D_{w} F^{\alpha, \lambda}(w)$ is invertible at $w=w^{\alpha, \lambda}$, and the desired conclusion follows from the implicit function theorem.

Theorem 1.1 is an immediate consequence of Proposition 4.2 we just set $\omega^{\alpha, \lambda}=$ $\alpha G+w^{\alpha, \lambda}, \mathbf{u}^{\alpha, \lambda}=\alpha \mathbf{v}^{G}+\mathbf{v}^{\alpha, \lambda}$ (where $\mathbf{v}^{\alpha, \lambda}$ is the velocity field obtained from $w^{\alpha, \lambda}$ by the Biot-Savart law), and $K_{0}=\left(4 K_{1} K_{5}\right)^{-1}$. By construction, $\omega^{\alpha, \lambda}$ is a stationary solution of (6) satisfying the conclusions of the theorem.

In the rest of this section, we establish a few additional properties of the asymmetric $\operatorname{vortex} \omega^{\alpha, \lambda}$ :

1) Expansion in $\lambda$. There exists $K_{6}>0$ such that, for all $(\alpha, \lambda) \in \mathbf{R} \times\left[0, \lambda_{0}\right]$ :

$$
\left\|\omega^{\alpha, \lambda}-\alpha G-\lambda w_{\alpha}\right\|_{Y} \leq \frac{K_{6}|\alpha| \lambda^{2}}{1+|\alpha|}
$$

Indeed, using the notations of Proposition 4.2 we have $w^{\alpha, \lambda} \in B_{r}$ with $r=4 K_{3}^{\alpha} \lambda$. As $w^{\alpha, \lambda}$ is a solution of (32), we obtain

$$
\left\|w^{\alpha, \lambda}-\lambda w_{\alpha}\right\|_{Y} \leq K_{1} K_{5} r^{2}+K_{2} \lambda r \leq \frac{K_{6}|\alpha| \lambda^{2}}{1+|\alpha|}
$$

2) Large Reynolds number asymptotics. Combining (37) and Proposition [3.4, we find for $|\alpha| \geq 1$ :

$$
\left\|\frac{1}{\alpha} \omega^{\alpha, \lambda}-G-\frac{\lambda}{\alpha} w_{\infty}\right\|_{Y} \leq \frac{K_{6} \lambda^{2}}{|\alpha|}+\frac{K_{4} \lambda}{|\alpha|^{2}} .
$$

In agreement with [11, we see that the leading correction to the Gaussian profile $G$ is $(\lambda / \alpha) w_{\infty}$, and that the higher order corrections are proportional to $\lambda^{2} / R$ and $\lambda / R^{2}$, where $R=|\alpha|$ is the Reynolds number.

3) Small Reynolds number asymptotics. Since $\mathcal{L} \mathcal{M} G=\mathcal{L}^{-1} \mathcal{M} G=-\mathcal{M} G$, it follows from (13) that $w_{\alpha}=\alpha \mathcal{M} G+\alpha^{2}(\mathcal{L}-\alpha \Lambda)^{-1} \Lambda \mathcal{M} G$. Replacing into (37) and using Proposition 2.1, we obtain for $|\alpha| \leq 1$ :

$$
\left\|\omega^{\alpha, \lambda}-\alpha G-\lambda \alpha \mathcal{M} G\right\|_{Y} \leq K_{6}|\alpha| \lambda^{2}+K_{1} \alpha^{2} \lambda\|\Lambda \mathcal{M} G\|_{X}
$$

In fact, if we proceed as in ([8, Section 2), this result can be improved as follows: there exists $K_{7}>0$ such that, for $|\alpha| \leq 1$,

$$
\left\|\omega^{\alpha, \lambda}-\alpha G_{\lambda}\right\|_{Y} \leq K_{7} \alpha^{2} \lambda
$$

where

$$
G_{\lambda}(x)=\frac{\sqrt{1-\lambda^{2}}}{4 \pi} \exp \left(-\frac{1+\lambda}{4} x_{1}^{2}-\frac{1-\lambda}{4} x_{2}^{2}\right), \quad x \in \mathbf{R}^{2} .
$$

Remark that $(\mathcal{L}+\lambda \mathcal{M}) G_{\lambda}=0$ and $\int_{\mathbf{R}^{2}} G_{\lambda} \mathrm{d} x=1$. Since $G_{\lambda}=G+\lambda \mathcal{M} G+\mathcal{O}\left(\lambda^{2}\right)$, we see that (39) is compatible with (40). 
4) Smoothness in $x$. Standard elliptic estimates imply that $\omega^{\alpha, \lambda}(x)$ is a smooth function of $x \in \mathbf{R}^{2}$ for any $(\alpha, \lambda) \in \mathbf{R} \times\left[0, \lambda_{0}\right]$. Indeed, since $\omega^{\alpha, \lambda}$ is a stationary solution of ([6), we have

$$
\omega^{\alpha, \lambda}=(\mathcal{L}+\lambda \mathcal{M})^{-1} \nabla \cdot\left(\mathbf{u}^{\alpha, \lambda} \omega^{\alpha, \lambda}\right) .
$$

It is not difficult to prove that the linear operator $(\mathcal{L}+\lambda \mathcal{M})^{-1} \nabla$ is regularizing, hence a bootstrap argument shows that $\omega^{\alpha, \lambda} \in H^{k}\left(\mathbf{R}^{2}\right)$ for all $k \in \mathbf{N}$. One can also prove that all derivatives decay rapidly at infinity, so that $\omega^{\alpha, \lambda} \in \mathcal{S}\left(\mathbf{R}^{2}\right)$.

5) Positivity. It follows from the parabolic Maximum Principle [15 that $\omega^{\alpha, \lambda}(x)>0$ for all $x \in \mathbf{R}^{2}$ if $\alpha>0$. (Similarly, $\omega^{\alpha, \lambda}(x)<0$ if $\alpha<0$, and we already know from (39) that $\omega^{\alpha, \lambda} \equiv 0$ if $\alpha=0$.) Indeed, arguing as in the symmetric case $\lambda=0$, it is not difficult to show that, for any initial data $\omega_{0} \in L^{1}\left(\mathbf{R}^{2}\right) \cap C^{0}\left(\mathbf{R}^{2}\right)$, Eq. (6) has a unique global solution $\omega \in C^{0}\left([0,+\infty), L^{1}\left(\mathbf{R}^{2}\right)\right)$ which satisfies $\int_{\mathbf{R}^{2}} \omega(x, t) \mathrm{d} x=\int_{\mathbf{R}^{2}} \omega_{0} \mathrm{~d} x$ for all $t \geq 0$. If $\omega_{0} \geq 0$ and $\omega_{0}$ is not identically zero, the Maximum Principle implies that $\omega(x, t)>0$ for all $x \in \mathbf{R}^{2}$ and all $t>0$, see e.g. ([7], Section 2.3). Conversely, if $\omega_{0}$ has non-constant sign, the norm $\|\omega(\cdot, t)\|_{L^{1}}$ is strictly decreasing in time (for $t>0$ sufficiently small), see (7], Section 3.1). Since $\omega^{\alpha, \lambda} \in L^{1}\left(\mathbf{R}^{2}\right) \cap C^{0}\left(\mathbf{R}^{2}\right)$ is a stationary solution of (6) satisfying $\int_{\mathbf{R}^{2}} \omega^{\alpha, \lambda} \mathrm{d} x=\alpha$, the properties above imply that $\omega^{\alpha, \lambda}(x)>0$ for all $x \in \mathbf{R}^{2}$ if $\alpha>0$.

\section{$5 \quad$ Stability of asymmetric vortices}

In this final section, we show that the asymmetric Burgers vortex $\omega^{\alpha, \lambda}$ constructed in Section 4 is a stable solution of (6) with respect to perturbations in $X$, provided $\lambda>0$ is sufficiently small. Fix $\alpha \in \mathbf{R}, \lambda \in\left[0, \lambda_{0}\right]$, and consider solutions of (6) of the form $\omega=\omega^{\alpha, \lambda}+\tilde{\omega}, \mathbf{u}=\mathbf{u}^{\alpha, \lambda}+\tilde{\mathbf{u}}$, where $\tilde{\mathbf{u}}$ is the velocity field obtained from $\tilde{\omega}$ by the BiotSavart law (3). Then $\tilde{\omega}$ satisfies the equation

$$
\partial_{t} \tilde{\omega}+\mathbf{u}^{\alpha, \lambda} \cdot \nabla \tilde{\omega}+\tilde{\mathbf{u}} \cdot \nabla \omega^{\alpha, \lambda}+\tilde{\mathbf{u}} \cdot \nabla \tilde{\omega}=(\mathcal{L}+\lambda \mathcal{M}) \tilde{\omega} .
$$

If we further decompose $\omega^{\alpha, \lambda}=\alpha G+w^{\alpha, \lambda}, \mathbf{u}^{\alpha, \lambda}=\alpha \mathbf{v}^{G}+\mathbf{v}^{\alpha, \lambda}$, this equation becomes

$$
\partial_{t} \tilde{\omega}+\tilde{\mathbf{u}} \cdot \nabla \tilde{\omega}=\left(\mathcal{L}+\lambda \mathcal{M}-\alpha \Lambda-\mathcal{N}^{\alpha, \lambda}\right) \tilde{\omega},
$$

where $\Lambda$ is defined in (11) and $\mathcal{N}^{\alpha, \lambda}$ is the integro-differential operator defined by

$$
\mathcal{N}^{\alpha, \lambda} \tilde{\omega}=\mathbf{v}^{\alpha, \lambda} \cdot \nabla \tilde{\omega}+\tilde{\mathbf{u}} \cdot \nabla w^{\alpha, \lambda} .
$$

It is easy to show that the Cauchy problem for (41) or (42) is locally well-posed in the space $X$. In the symmetric case $\lambda=0$, this is proved in [6] using a larger function space (with polynomial instead of Gaussian weight), and the same arguments apply here with straightforward modifications. Our goal is to control the behavior of the solutions of (42) in a neighborhood of the origin. An energy estimate yields the following result:

Proposition 5.1 There exist positive constants $K_{8}, K_{9}$ (independent of $\alpha$ and $\lambda$ ) such that, for any $\delta \in(0,1)$, any solution solution of (42) in $X$ satisfies

$$
\frac{\mathrm{d}}{\mathrm{d} t}\|\tilde{\omega}(t)\|_{X}^{2} \leq-(1-\delta)\|\tilde{\omega}(t)\|_{X}^{2}
$$

whenever $K_{8} \lambda+K_{9}\|\tilde{\omega}(t)\|_{X} \leq \delta$. 
Proof: From (42) we obtain

$$
\frac{1}{2} \frac{\mathrm{d}}{\mathrm{d} t}\|\tilde{\omega}\|_{X}^{2}=\left(\tilde{\omega},\left(\mathcal{L}+\lambda \mathcal{M}-\alpha \Lambda-\mathcal{N}^{\alpha, \lambda}\right) \tilde{\omega}\right)_{X}-(\tilde{\omega}, \tilde{\mathbf{u}} \cdot \nabla \tilde{\omega})_{X}
$$

To simplify the subsequent expressions, we define $f=G^{-1 / 2} \tilde{\omega}$, so that $\|\tilde{\omega}\|_{X}=\|f\|_{L^{2}}$, and we introduce the quadratic form

$$
E(f)=\int_{\mathbf{R}^{2}}\left(|\nabla f|^{2}+\frac{|x|^{2}}{16} f^{2}\right) \mathrm{d} x
$$

Obviously $\|f\|_{L^{2}}^{2} \leq C E(f)$, and we have $\|f\|_{L^{4}}^{2} \leq C\|\nabla f\|_{L^{2}}^{3 / 2}\|x f\|_{L^{2}}^{1 / 2} \leq C E(f)$, see [2].

Proceeding as in (17), we obtain for any $\delta \in(0,1)$ :

$$
(\tilde{\omega}, \mathcal{L} \tilde{\omega})_{X}=-(f, L f)_{L^{2}} \leq-\frac{1-\delta}{2}\|f\|_{L^{2}}^{2}-\frac{\delta}{2} E(f)
$$

(The bound (17) was the particular case $\delta=1 / 2$.) Next, since $\mathcal{M}=\frac{1}{2}\left(x_{1} \partial_{1}-x_{2} \partial_{2}\right)$ and $G^{-1 / 2} \nabla \tilde{\omega}=\nabla f-\frac{x}{4} f$, we find

$$
(\tilde{\omega}, \mathcal{M} \tilde{\omega})_{X} \leq\|x \tilde{\omega}\|_{X}\|\nabla \tilde{\omega}\|_{X}=\|x f\|_{L^{2}}\left\|\nabla f-\frac{x}{4} f\right\|_{L^{2}} \leq C E(f) .
$$

Moreover, as was observed in the proof of Lemma 2.3. the operator $\Lambda$ is skew-symmetric in $X$, hence $(\tilde{\omega}, \Lambda \tilde{\omega})_{X}=0$.

We now bound the nonlinear term in (43). Integrating by parts and using the relation $\nabla G^{-1}=\frac{x}{2} G^{-1}$, we obtain

$$
(\tilde{\omega}, \tilde{\mathbf{u}} \cdot \nabla \tilde{\omega})_{X}=\int_{\mathbf{R}^{2}} G^{-1} \tilde{\omega}(\tilde{\mathbf{u}} \cdot \nabla \tilde{\omega}) \mathrm{d} x=-\frac{1}{4} \int_{\mathbf{R}^{2}} G^{-1}(x \cdot \tilde{\mathbf{u}}) \tilde{\omega}^{2} \mathrm{~d} x .
$$

Applying Hölder's inequality, we find

$$
\left|(\tilde{\omega}, \tilde{\mathbf{u}} \cdot \nabla \tilde{\omega})_{X}\right| \leq \frac{1}{4} \int_{\mathbf{R}^{2}}|\tilde{\mathbf{u}}|\left|x f\left\|f \mid \mathrm{d} x \leq \frac{1}{4}\right\| \tilde{\mathbf{u}}\left\|_{L^{4}}\right\| f\left\|_{L^{4}}\right\| x f\left\|_{L^{2}} \leq C\right\| \tilde{\omega} \|_{X} E(f),\right.
$$

where we have used the bound $\|\tilde{\mathbf{u}}\|_{L^{4}} \leq C\|\tilde{\omega}\|_{L^{4 / 3}} \leq C\|\tilde{\omega}\|_{X}$, see (20) and (21).

It remains to bound $\left(\tilde{\omega}, \mathcal{N}^{\alpha, \lambda} \tilde{\omega}\right)_{X}=\left(\tilde{\omega}, \mathbf{v}^{\alpha, \lambda} \cdot \nabla \tilde{\omega}\right)_{X}+\left(\tilde{\omega}, \tilde{\mathbf{u}} \cdot \nabla w^{\alpha, \lambda}\right)_{X}$. The first term in this sum can be estimated in the same way as the nonlinear term above, namely $\left|\left(\tilde{\omega}, \mathbf{v}^{\alpha, \lambda} \cdot \nabla \tilde{\omega}\right)_{X}\right| \leq C\left\|w^{\alpha, \lambda}\right\|_{X} E(f)$. For the second term, we argue differently:

$$
\begin{aligned}
\left|\left(\tilde{\omega}, \tilde{\mathbf{u}} \cdot \nabla w^{\alpha, \lambda}\right)_{X}\right| & \leq \int_{\mathbf{R}^{2}} G^{-1}\left|\tilde{\omega}\|\tilde{\mathbf{u}}\| \nabla w^{\alpha, \lambda}\right| \mathrm{d} x \\
& \leq\left\|\nabla w^{\alpha, \lambda}\right\|_{X}\|\tilde{\mathbf{u}}\|_{L^{4}}\|f\|_{L^{4}} \leq C\left\|w^{\alpha, \lambda}\right\|_{Y} E(f),
\end{aligned}
$$

where we have used $\|\tilde{\mathbf{u}}\|_{L^{4}} \leq C\|\tilde{\omega}\|_{L^{4 / 3}} \leq C\|f\|_{L^{2}} \leq C E(f)^{1 / 2}$. Thus, using Proposition 4.2 to bound $w^{\alpha, \lambda}$, we conclude that $\left|\left(\tilde{\omega}, \mathcal{N}^{\alpha, \lambda} \tilde{\omega}\right)_{X}\right| \leq C\left\|w^{\alpha, \lambda}\right\|_{Y} E(f) \leq C \lambda E(f)$. Summarizing, we have shown that

$$
\frac{1}{2} \frac{\mathrm{d}}{\mathrm{d} t}\|\tilde{\omega}\|_{X}^{2} \leq-\frac{1-\delta}{2}\|\tilde{\omega}\|_{X}^{2}+\frac{1}{2}\left(K_{8} \lambda+K_{9}\|\tilde{\omega}\|_{X}-\delta\right) E\left(G^{-1 / 2} \tilde{\omega}\right)
$$


for some constants $K_{8}, K_{9}$ independent of $\alpha, \lambda$ and of the solution $\tilde{\omega}$ of (42). This concludes the proof.

Theorem 1.2 is now a direct consequence of Proposition 5.1. Given $\mu \in(0,1 / 2)$, it suffices to choose $\lambda_{1} \in\left(0, \lambda_{0}\right]$ such that $K_{8} \lambda_{1} \leq \delta / 2$ and $\varepsilon>0$ such that $K_{9} \varepsilon<\delta / 2$, where $\delta=1-2 \mu$. If $\tilde{\omega}_{0} \in X$ satisfies $\left\|\tilde{\omega}_{0}\right\|_{X} \leq \varepsilon$ and if $\tilde{\omega} \in C^{0}\left(\left[0, T^{*}\right), X\right)$ denotes the (maximal) solution of (42) with initial data $\tilde{\omega}_{0}$, we define

$$
T=\sup \left\{t \in\left(0, T^{*}\right) \mid K_{9}\|\tilde{\omega}(s)\|_{X} \leq \delta / 2 \text { for } 0 \leq s \leq t\right\} \in\left(0, T^{*}\right] .
$$

If $T<+\infty$ then Proposition 5.1 implies that $\|\tilde{\omega}(t)\|_{X} \leq e^{-\mu t}\left\|\tilde{\omega}_{0}\right\|_{X}$ for $t \in[0, T]$, hence $K_{9}\|\tilde{\omega}(t)\|_{X} \leq K_{9} \varepsilon<\delta / 2$ for $t \in[0, T]$, which contradicts the definition of $T$. Thus, we must have $T=T^{*}=+\infty$. This means that the solution $\tilde{\omega}$ is globally defined, and by Proposition $5.1\|\tilde{\omega}(t)\|_{X} \leq e^{-\mu t}\left\|\tilde{\omega}_{0}\right\|_{X}$ for all $t \geq 0$.

We conclude with a few remarks on the basin of attraction of the asymmetric vortex and the decay rate in time of the perturbations:

a) Proposition 5.1 shows that the decay rate in time of pertubations of the asymmetric vortex $\omega^{\alpha, \lambda}$ is bounded from below by $\mu=\frac{1}{2}\left(1-K_{8} \lambda\right)=\frac{1}{2}-\mathcal{O}(\lambda)$, uniformly in $\alpha \in \mathbf{R}$. This is consistent with the information we have on the spectrum of the linearized operator

$$
\mathcal{L}^{\alpha, \lambda}=\mathcal{L}+\lambda \mathcal{M}-\alpha \Lambda-\mathcal{N}^{\alpha, \lambda}
$$

acting on the space $X$. Indeed, differentiating the identity $(\mathcal{L}+\lambda \mathcal{M}) \omega^{\alpha, \lambda}=\mathbf{u}^{\alpha, \lambda} \cdot \nabla \omega^{\alpha, \lambda}$ with respect to $x_{1}$ and $x_{2}$ we obtain

$$
\mathcal{L}^{\alpha, \lambda}\left(\partial_{1} \omega^{\alpha, \lambda}\right)=-\frac{1+\lambda}{2}\left(\partial_{1} \omega^{\alpha, \lambda}\right), \quad \mathcal{L}^{\alpha, \lambda}\left(\partial_{2} \omega^{\alpha, \lambda}\right)=-\frac{1-\lambda}{2}\left(\partial_{2} \omega^{\alpha, \lambda}\right) .
$$

In particular, since $\partial_{2} \omega^{\alpha, \lambda} \in X$, we see that $-\frac{1-\lambda}{2}$ is always an eigenvalue of $\mathcal{L}^{\alpha, \lambda}$, hence $\mu \leq \frac{1-\lambda}{2}$. Numerical calculations by Prochazka and Pullin [14] seem to indicate that $-\frac{1-\lambda}{2}$ is always the largest eigenvalue of $\mathcal{L}^{\alpha, \lambda}$ in $X$ (for any $\lambda$ ). If this was true our arguments could be extended to prove existence and stability of asymmetric Burgers vortices for all $\lambda \in[0,1)$. Put another way, our current limitation on the range of the asymmetry parameter is only due to the fact that we do not know how to control the eigenvalues of $\mathcal{L}^{\alpha, \lambda}$ (except of course for small $\lambda$ ).

b) A remarkable feature of our stability result (Theorem 1.2) is that it holds uniformly for all $\alpha \in \mathbf{R}$. In particular, this implies a uniform upper bound on the eigenvalues of the linearized operator $\mathcal{L}^{\alpha, \lambda}$. This is definitely compatible with the numerical observations of Prochazka and Pullin [13], but these calculations suggest that our result is perhaps not optimal for large Reynolds numbers. According to 13 we expect that the eigenvalues that are not frozen by symmetries have a real part that converges to $-\infty$ as $|\alpha| \rightarrow \infty$, which could imply a faster decay rate $\mu$ and a larger basin of attraction $\varepsilon$ for large Reynolds numbers. A mathematical understanding of these numerical observations is still lacking.

c) We chose to consider perturbations $\tilde{\omega}$ in the weighted space $X$ because of the "miraculous" fact that the operator $\Lambda$ is skew-symmetric in that space. This is why Proposition 5.1 holds uniformly for all $\alpha \in \mathbf{R}$. However the space $X$ is relatively small since its elements 
are forced to decay rapidly at infinity in space. Extending the methods developed in [7] for the symmetric case $\lambda=0$, it is not difficult to show that the asymmetric Burgers vortices are also stable with respect to perturbations in weighted $L^{2}$ spaces with polynomial (instead of Gaussian) weight. The decay rate in time of the perturbations is still uniform in $\alpha$, but the size of the basin of attraction is a priori not.

d) In the symmetric case $\lambda=0$ it is shown in [7] that the Burgers vortex $\alpha G$ is the unique stationary solution of (6) such that $\omega \in L^{1}\left(\mathbf{R}^{2}\right)$ and $\int_{\mathbf{R}^{2}} \omega \mathrm{d} x=\alpha$. Moreover, any solution $\omega \in C^{0}\left([0,+\infty), L^{1}\left(\mathbf{R}^{2}\right)\right)$ of (66) such that $\int_{\mathbf{R}^{2}} \omega(x, t) \mathrm{d} x=\alpha$ converges to $\alpha G$ in $L^{1}\left(\mathbf{R}^{2}\right)$ as $t \rightarrow+\infty$. We do not know if such global results can be extended to the nonsymmetric case $\lambda>0$.

Acknowledgements. A part of this work was completed when $\mathrm{CEW}$ was a visitor at Institut Fourier, Université de Grenoble I, whose hospitality is gratefully acknowledged. The research of CEW is supported in part by the NSF through grant DMS-0405724, and the work of ThG is supported by the ACI "Structure and dynamics of nonlinear waves" of the French Ministry of Research.

\section{References}

[1] J.M. Burgers. A mathematical model illustrating the theory of turbulence. Adv. Appl. Mech., 1:171-199, 1948.

[2] L. Caffarelli, R. Kohn, and L. Nirenberg. First order interpolation inequalities with weights. Compositio Math., 53(3):259-275, 1984.

[3] E. A. Coddington and N. Levinson. Theory of ordinary differential equations. McGraw-Hill Book Company, Inc., New York-Toronto-London, 1955.

[4] D. G. Crowdy. A note on the linear stability of Burgers vortex. Stud. Appl. Math., 100(2):107-126, 1998.

[5] C. Eloy and S. Le Dizès. Three-dimensional instability of Burgers and Lamb-Oseen vortices in a strain field. J. Fluid Mech., 378:145-166, 1999.

[6] Th. Gallay and C. E. Wayne. Invariant manifolds and the long-time asymptotics of the Navier-Stokes and vorticity equations on $\mathbb{R}^{2}$. Arch. Ration. Mech. Anal., 163(3):209-258, 2002.

[7] Th. Gallay and C. E. Wayne. Global stability of vortex solutions of the twodimensional Navier-Stokes equation. Comm. Math. Phys., 255(1):97-129, 2005.

[8] Th. Gallay and C. E. Wayne. Three-dimensional stability of Burgers vortices : the case of low Reynolds number. Preprint, 2005.

[9] J. Jiménez, H. K. Moffatt, and C. Vasco. The structure of the vortices in freely decaying two-dimensional turbulence. J. Fluid Mech., 313:209-222, 1996. 
[10] E. H. Lieb and M. Loss. Analysis, volume 14 of Graduate Studies in Mathematics. American Mathematical Society, Providence, RI, 1997.

[11] H. K. Moffatt, S. Kida, and K. Ohkitani. Stretched vortices - the sinews of turbulence; large-Reynolds-number asymptotics. J. Fluid Mech., 259:241-264, 1994.

[12] L. Nirenberg. On elliptic partial differential equations. Ann. Scuola Norm. Sup. Pisa (3), 13:115-162, 1959.

[13] A. Prochazka and D. I. Pullin. On the two-dimensional stability of the axisymmetric Burgers vortex. Phys. Fluids, 7(7):1788-1790, 1995.

[14] A. Prochazka and D. I. Pullin. Structure and stability of non-symmetric Burgers vortices. J. Fluid Mech., 363:199-228, 1998.

[15] M. H. Protter and H. F. Weinberger. Maximum principles in differential equations. Prentice-Hall Inc., Englewood Cliffs, N.J., 1967.

[16] M. Reed and B. Simon. Methods of modern mathematical physics. I. Academic Press Inc., New York, second edition, 1980. Functional analysis.

[17] A. C. Robinson and P. G. Saffman. Stability and structure of stretched vortices. Stud. Appl. Math., 70(2):163-181, 1984.

[18] M. Rossi and S. Le Dizès. Three-dimensional temporal spectrum of stretched vortices. Phys. Rev. Lett., 78:2567-2569, 1997.

[19] P. J. Schmid and M. Rossi. Three-dimensional stability of a Burgers vortex. J. Fluid Mech., 500:103-112, 2004.

[20] E. Stein. Singular integrals and differentiability properties of functions. Princeton University Press, Princeton, N.J., 1970. Princeton Mathematical Series, No. 30.

[21] G. I. Taylor. Production and dissipation of vorticity in a turbulent fluid. Proc. $R$. Soc. Lond. A, 164:15-23, 1938. 